Copyright ( 2013 IEEE. Personal use of this material is permitted. Permission from IEEE must be obtained for all other uses, in any current or future media, including reprinting/republishing this material for advertising or promotional purposes, creating new collective works, for resale or redistribution to servers or lists, or reuse of any copyrighted component of this work in other works. 


\section{Multi-Scale Control}

\section{Improved Technique to Overcome Time-Delay Limitation}

\author{
Jobrun Nandong \\ Department of Chemical Engineering \\ Curtin University Sarawak \\ 98009 Miri, Sarawak, Malaysia \\ jobrun.n@curtin.edu.my
}

\author{
Zhuquan Zang \\ Department of Electrical and Computer Engineering \\ Curtin University Sarawak \\ 98009 Miri, Sarawak, Malaysia \\ zqzang@curtin.edu.my
}

\begin{abstract}
This paper presents a general multi-scale control scheme which can be used to control processes with significant time-delays. The salient feature of the multi-scale control scheme is to decompose a given plant into a sum of basic factors or modes. An individual sub-controller is specifically designed to control each of the plant modes and subsequently, an overall multi-scale controller is synthesized via combining all of the subcontrollers in a manner to enhance cooperation among these different plant modes. Numerical examples show that the multiscale control scheme can provide improved performance and robustness over the conventional single-loop PID and Smith predictor schemes.
\end{abstract}

Keywords-PID; Smith Predictor;Time-Delay Process; MultiScale Control

\section{INTRODUCTION}

One of the most common factors limiting the closed-loop performance in many real systems is time-delay, which imposes an upper limit on control performance [1]. To maintain stability, it is often required to detune the conventional single-loop PID control. Since the traditional PID controllers are normally ineffective in controlling time-delay systems, the most prevalent approach to controlling this type of systems is usually based on the Smith predictor [2]. The Smith predictor structure is as shown in Figure 1.

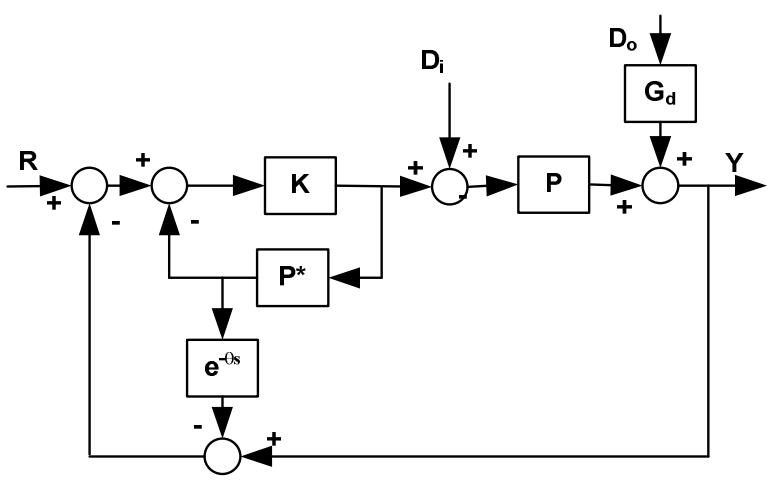

Figure 1. Smith Predictor scheme for time-delay system
Based on Figure 1, assuming that $P=P^{*}$, the closed-loop transfer function from the setpoint $R$ to the controlled output $Y$ is given by

$$
\frac{Y(s)}{R(s)}=\frac{K(s) P^{*}(s) e^{-\theta s}}{1+K(s) P^{*}(s)}
$$

where $P^{*}$ denotes the delay-free nominal model, $P$ the actual plant, $D_{i}$ the input disturbance, $D_{o}$ the output disturbance, $G_{d}$ the output disturbance transfer function; $K$ the controller.

As the Smith predictor design requires model of a given plant, the stability of the scheme is often susceptible to modeling error [3]. The necessary and sufficient conditions for the practical stability and robust stability for Smith predictor scheme is given in [4]. A number of variants of the Smith predictor have been developed over the years, e.g. [5]-[7].

In this paper, we present a new approach to control timedelay systems called the multi-scale control (MSC) scheme. The basic idea of the proposed MSC scheme is to decompose a given plant into a sum of basic factors or modes with distinct speed of responses. An individual sub-controller is then specifically designed to control each of the plant modes. Subsequently, an overall multi-scale controller is synthesized by combining all of the sub-controllers in a manner to enhance good cooperation among the different plant modes - this is the essential feature to improve control performance and robustness. Note that, in a conventional single-loop (PID) control scheme, it can be interpreted that a single controller is often designed based on the slow dominant modes, i.e. the fast modes are often ignored. Thus, this single controller might not lead to good cooperation among the different plant modes, which subsequently causes poor control performance and even robustness.

The rest of the paper is structured as follows. In Section II, the concept of multi-scale control scheme is presented alongside with a general design procedure. In Section III, numerical examples are presented. Section IV highlights some conclusions of the work. 


\section{Multi-ScAle CONTROL Scheme}

\section{A. Multi-scale Plant Decomposition}

Assume that a plant $P$ to be controlled is given by a rational transfer function. By applying partial fraction expansion, the plant can be decomposed into a sum of factors as follows:

$$
P(s)=P_{0}(s)+P_{1}(s)+\ldots+P_{n}(s)
$$

For a system with time delay, the delay part can often be approximated using the first order (1/1) Padé formula [8]:

$$
e^{-\theta s} \approx G_{t d}(s)=\frac{1-0.5 \theta s}{1+0.5 \theta s}
$$

Hence, the overall plant model can be written as follows:

$$
P(s)=P^{*}(s) e^{-\theta s} \approx P^{*}(s) G_{t d}(s)
$$

\section{B. Realization of Multi-Scale Control Scheme}

Figure 2 shows the block diagram of 3-layer multi-scale feedback control scheme for a given plant $P$ that can be decomposed into 3 factors $\left(P=P_{0}+P_{1}+P_{2}\right)$. Here, $W_{i}$ is called the multi-scale predictors; $K_{i}$ the multi-scale subcontrollers; $P_{c}$ the augmented overall plant transfer function. The outermost sub-controller $\left(K_{0}\right)$ corresponds to the factor with the slowest dynamic $\left(P_{0}\right)$ and $K_{2}$ corresponds to the fastest dynamic $\left(P_{2}\right)$.

Note that, the closed-loop transfer functions for the inner layers are given by:

$$
\begin{gathered}
G_{1}(s)=\frac{K_{1}(s)}{1+G_{2}(s) K_{1}(s) W_{1}(s)} \\
G_{2}(s)=\frac{K_{2}(s)}{1+K_{2}(s) W_{2}(s)}
\end{gathered}
$$

The augmented overall plant transfer function

$$
P_{c}(s)=G_{1}(s) G_{2}(s) P(s)
$$

For a plant that can be decomposed into a sum of $n+1$ factors such as $P=P_{0}+P_{1}+P_{2}+\ldots+P_{n}$; there are $n$ inner control loops (or sub-controllers) corresponding to the $n$ inner-layer factors. The notations $P_{0}$ and $P_{n}$ represent the slowest and the fastest factors, respectively.
Furthermore, for a plant with $n+1$ factors, we can further extend the 3-layer block diagram shown in Figure 2 into $n+1$ layer block diagram. We can derive a vector of closed-loop transfer functions $\boldsymbol{G}=\left[\begin{array}{lllll}G_{1} & G_{2} & \cdots & G_{n}\end{array}\right]^{T}$ for the $\boldsymbol{n}$ inner layers as follows:

$$
\boldsymbol{G}=\left[\begin{array}{c}
G_{1}(s) \\
G_{2}(s) \\
\vdots \\
G_{n-1}(s) \\
G_{n}(s)
\end{array}\right]=\left[\begin{array}{c}
\frac{K_{1}(s)}{1+K_{1}(s) W_{1}(s)\left\{\prod_{j=2}^{n} G_{j}(s)\right\}} \\
\frac{K_{2}(s)}{1+K_{2}(s) W_{2}(s)\left\{\prod_{j=3}^{n} G_{j}(s)\right\}} \\
\vdots \\
\frac{K_{n-1}(s)}{1+K_{n-1}(s) W_{n-1}(s)\left\{\prod_{j=n}^{n} G_{j}(s)\right\}} \\
\frac{K_{n}(s)}{1+K_{n}(s) W_{n}(s)}
\end{array}\right]
$$

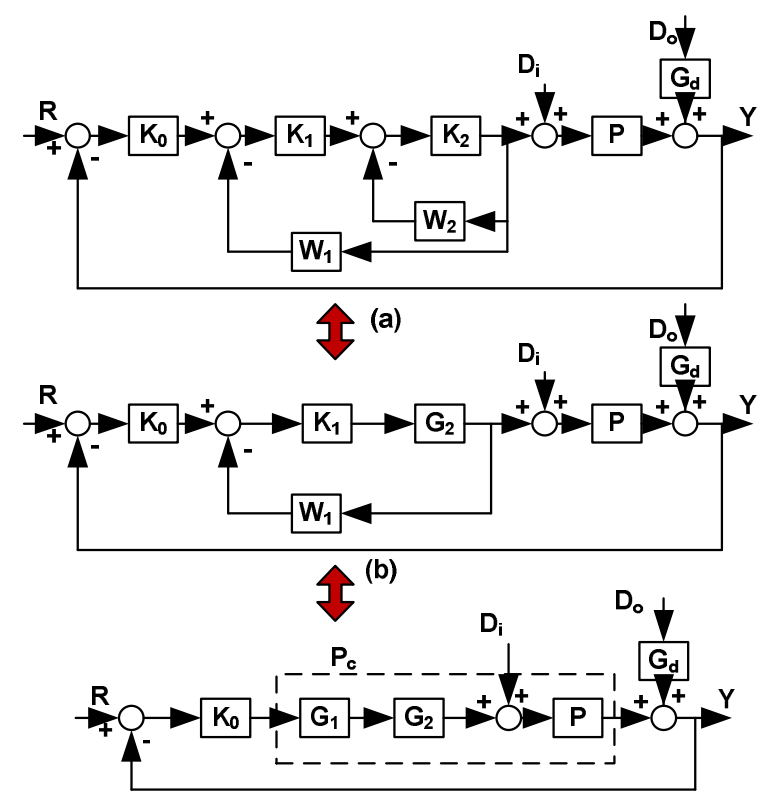

(c)

Figure 2. Block diagram of 3-layer multi-scale feedback control scheme: (a) full 3-loop, (b) reduced 2-loop and (c) reduced single-loop block diagrams.

The sub-controllers are consecutively designed starting from the innermost layer $K_{n}$ followed by $K_{n-1}, K_{n-2}, \ldots, K_{1}$. It is often helpful to derive the following vector of transfer functions $\mathcal{H} \in R^{n-1}$ in order to design the sub-controllers: 


$$
\mathcal{H}=\left[\begin{array}{c}
H_{1}(s) \\
H_{2}(s) \\
\vdots \\
H_{n-2}(s) \\
H_{n-1}(s)
\end{array}\right]=\left[\begin{array}{l}
\prod_{j=2}^{n}\left[G_{j}(s)\right] W_{1}(s) \\
\prod_{j=3}^{n}\left[G_{j}(s)\right] W_{2}(s) \\
\vdots \\
\prod_{j=n-1}^{n}\left[G_{j}(s)\right] W_{n-2}(s) \\
\prod_{j=n}^{n}\left[G_{j}(s)\right] W_{n-1}(s)
\end{array}\right]
$$

Once all the inner layer sub-controllers have been designed, the outermost sub-controller $\left(K_{0}\right)$ can be designed based on the augmented overall plant transfer function:

$$
P_{c}(s)=\prod_{j=1}^{n}\left[G_{j}(s)\right] P(s)
$$

To design $K_{0}$, it is normally assumed that $P=\bar{P}$ where $\bar{P}$ is the nominal model for the plant.

\section{Design Procedure}

Step 1: Decompose the plant (model) $P$ into a sum of factors by using partial fraction expansion as in (2).

Step 2: Design the innermost sub-controller $K_{n}$ based on $W_{n}=P_{n}$.

Step 3: Derive the transfer functions $G_{n}$ as in (8) and then $H_{n-1}$ as in (9).

Step 4: Design the sub-controller $K_{n-1}$ based on $H_{n-1}$. Repeat Steps 3 and 4 to obtain $K_{n-2}, K_{n-3}, \ldots, K_{1}$.

Step 6: Once all the inner sub-controllers have been designed, then derive the augmented overall plant transfer function $P_{c}$ as in (10). Finally, use $P_{c}$ to design $K_{0}$.

\section{Remark:}

- For the inner sub-controllers, it is often recommended to adopt a very simple controller algorithm (the proportional or P-only controller).

- As for the outermost sub-controller $K_{0}$, it is recommended to adopt a proportional-integral (PI) controller for reason of simple tuning.

- We can also adopt more complex controller algorithms especially for the outermost sub-controller if a desired performance cannot be met using the PI controller, e.g. a proportional-integral-derivative (PID) controller with a lag filter and Linear-Quadratic-Gaussian (LQG) controller.

\section{ILLUSTRATIVE EXAMPLES}

\section{Example 1}

Assume that the system to be controlled is given by the transfer function below:

$$
P(s)=\frac{2 e^{-(10+a) s}}{10 s+1}
$$

where $a$ represents plant/model mismatch or modeling error for the time-delay.

Furthermore, assume that the output disturbance is given by

$$
G_{d}=\frac{1}{5 s+1}
$$

Assuming that $a=0$, approximate the time-delay component in (11) using the 1/1 Padé formula (3). The nominal model can be written as follows:

$$
\bar{P}(s)=\frac{2}{(10 s+1)} \frac{(1-5 s)}{(1+5 s)}
$$

Next, we can decompose (13) into two factors using partial fraction expansion:

$$
\left[\begin{array}{l}
P_{0}(s) \\
P_{1}(s)
\end{array}\right]=\left[\begin{array}{l}
\frac{6}{10 s+1} \\
\frac{-4}{5 s+1}
\end{array}\right]
$$

For the synthesis of multi-scale control (MSC) scheme, we select the multi-scale predictor as $W_{1}=P_{1}$. The general procedure given in the previous section is adopted to synthesize the multi-scale controller.

The controller tuning is done via the automated tuning procedure based on the minimization of Integral Absolute Error (IAE) criterion, performed using the Matlab SISO Design Tool:

$$
K_{1}=-2.25
$$

Then, derive the closed-loop transfer function $G_{1}$ as in (9) for the case where $n=1$ ( 1 inner layer exists). Subsequently, derive the augmented overall plant transfer function $P_{c}=G_{1} P$ using the approximated plant model in (13). Finally, proceed with the outermost sub-controller $K_{0}$ design, where the application of automated tuning procedure for PI controller leads to:

$$
K_{0}(s)=\frac{-0.192(9 s+1)}{s}
$$


For performance comparison, two other control schemes are designed: (1) PI controller with Smith predictor and (2) conventional single-loop PID controller.

The control scheme employing a PI controller augmented with the Smith predictor (Figure 1) is designed based on the delay-free model of the plant. Note that, the time-delay component is ignored in the controller design. The application of Matlab SISO Design Tool (automated tuning):

$$
K_{P I}(s)=\frac{0.18(12 s+1)}{s}
$$

Next, the conventional single-loop PID controller with a lag filter is designed based on the approximated plant model as in (13). The following controller is obtained:

$$
K_{P I D}(s)=\frac{0.07\left(1+9.8 s+35 s^{2}\right)}{s}\left(\frac{1}{0.05 s+1}\right)
$$

Figure 3 shows the closed-loop responses of the 3 control schemes for the case when the model is exact in response to 1 unit step change in output disturbance. Notice that at the nominal condition, the multi-scale control scheme (MSC-PI/P) and the Smith predictor scheme (PI wt. Smith) exhibit almost the same performance in term of IAE value. Meanwhile, the standard single-loop PID controller shows more sluggish response in term of IAE value (13.7) than the other two control schemes.

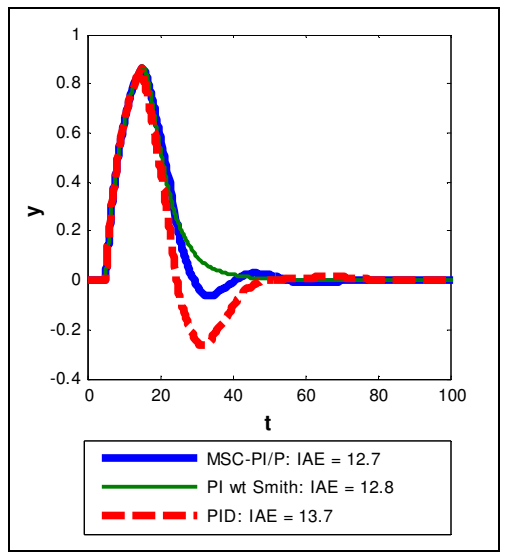

Figure 3. Disturbance rejection performance for zero model error: $a=0$

Figure 4 shows the comparative performances of the 3 control schemes in the presence of modelling error in timedelay (5 units). In this case, the PI with Smith Predictor control scheme is unstable. On the other hand, the MSC-PI/P and PID control schemes remain stable for this modeling error condition. However, the performance of the standard PID control scheme is badly degraded; its IAE increases from 13.7 to 99.9. It is worth highlighting that, the performance of the multi-scale control (MSC) scheme is more robust than that of the standard single-loop PID controller; its IAE only increases by about 1.7 times that at the nominal condition, i.e. it experiences much less performance degradation.

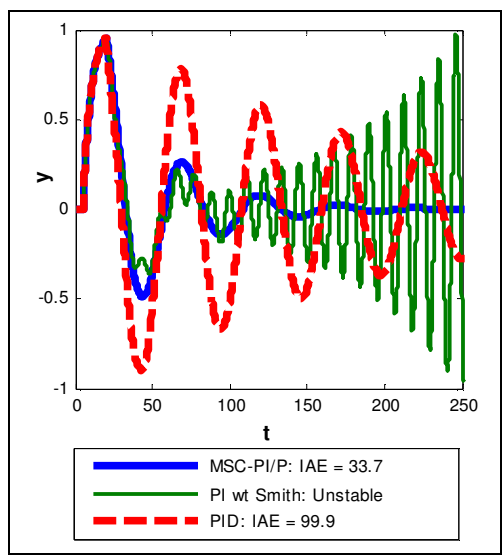

Figure 4. Disturbance rejection performance for $a=5$

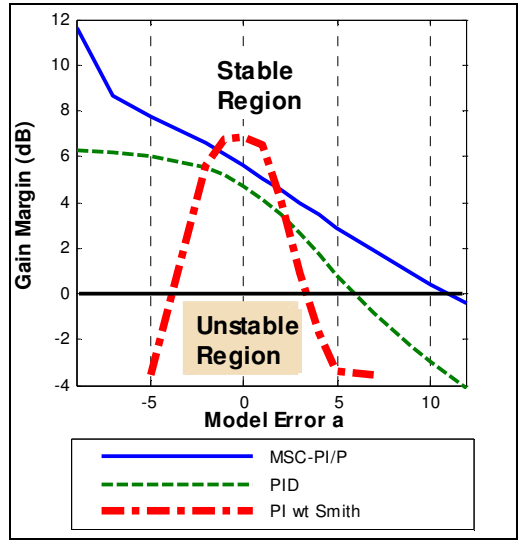

Figure 5. Gain margin vs. time-delay modeling error

Note that, we can calculate the gain margin for the control system using Nyquist plot. For the proposed MSC scheme, the gain margin is calculated based on the overall open-loop transfer function given by

$$
G_{O L}(s)=K_{0}(s) G_{1}(s) P(s)
$$

For the conventional single-loop PID controller, the overall open-loop transfer function

$$
G_{O L}(s)=K_{P I D}(s) P(s)
$$

And for the Smith predictor scheme, the overall open-loop transfer function is given by

$$
G_{O L}(s)=\frac{K_{P I}(s) P(s)}{1+K_{P I}(s)\left[P^{*}(s)-\bar{P}(s)\right]}
$$


The plant $P$ shown in (19)-(21) is as given by (11); $\bar{P}$ denotes the approximated model as in (13) after time-delay approximation using the $4^{\text {th }}$-order Padé formula; $P^{*}$ the plant model as in (11) assuming no time-delay component. The value of time-delay modeling error $a$ in (11) is varied while keeping all controllers fixed. Then, Nyquist plot is analyzed at each value of $a$ for gain margin.

Figure 5 displays the plot of gain margins for the 3 control schemes against the error in time-delay (negative sign indicates reduction in the actual time-delay and vice versa). Notice that, the gain margins for the MSC-PI/P and PID schemes increase with the reduction in the actual time-delay, which means that the control schemes will only become unstable when the actual time-delay increases beyond certain values above that of the nominal value. On the contrary, the PI with Smith predictor scheme is stable only within a narrow range of error in timedelay. From Figure 5, obviously we can notice that the MSC$\mathrm{PI} / \mathrm{P}$ is the most robust against the plant/model mismatch in time-delay where the proposed scheme will only become unstable when the increase in the actual time delay exceeds 11 units. Note that, the standard single-loop PID controller can only maintain stability for the increase in the actual time-delay up to 6 units. Note that, the PI with Smith predictor scheme is the least robust against the plant/model mismatch in timedelay.

Overall, based on this example we can draw a conclusion that it is possible to achieve a better closed-loop performance using the proposed multi-scale control scheme than the Smith predictor scheme for time-delay systems. It is worth noting that, for a system with dynamics dominated by long time-delay, the proposed scheme can be more robust against modeling error in time delay than the Smith predictor or the standard single-loop PID controller.

\section{Example 2}

Assume the system to be controlled is given by the second order plus time-delay (SOPDT) transfer function as follows:

$$
P(s)=\frac{0.8 e^{-(10+a) s}}{400 s^{2}+50 s+1}
$$

And let the output disturbance transfer function

$$
G_{d}(s)=\frac{-1}{15 s+1}
$$

Assuming that model error $a=0$, the application of $1 / 1$ Padé formula (3) yields an approximated model:

$$
\bar{P}(s)=\frac{0.8(1-5 s)}{\left(400 s^{2}+50 s+1\right)(1+5 s)}
$$

Application of partial fraction expansion to (24) leads to

$$
\bar{P}(s)=\frac{1.372}{40 s+1}-\frac{0.8}{10 s+1}+\frac{0.228}{5 s+1}
$$

where the plant factors are given by

$$
\left[\begin{array}{l}
P_{0}(s) \\
P_{1}(s) \\
P_{2}(s)
\end{array}\right]=\left[\begin{array}{c}
\frac{1.372}{40 s+1} \\
\frac{-0.8}{10 s+1} \\
\frac{0.228}{5 s+1}
\end{array}\right]
$$

The multi-scale predictors are selected as

$$
\left[\begin{array}{l}
W_{1}(s) \\
W_{2}(s)
\end{array}\right]=\left[\begin{array}{l}
P_{1}(s) \\
P_{2}(s)
\end{array}\right]
$$

The multi-scale control scheme is designed according to the procedure described in Section 2.3. All sub-controllers for the inner-loops are chosen as P-only controllers. Meanwhile, a PI controller is chosen for the outermost sub-controller. This results in the sub-controllers as given by

$$
\left[\begin{array}{l}
K_{0} \\
K_{1} \\
K_{2}
\end{array}\right]=\left[\begin{array}{c}
-0.0573\left(\frac{41 s+1}{s}\right) \\
-5.35 \\
4.67
\end{array}\right]
$$

As for the Smith predictor scheme, a PI controller is designed using (22), but ignoring the time-delay component:

$$
K_{P I}(s)=\frac{0.29(22 s+1)}{s}
$$

And for the single-loop feedback control scheme, a PID controller is augmented with a lag filter. The controller is designed based on the approximated model (24):

$$
K_{P I D}(s)=\frac{0.06\left(1+52 s+425.6 s^{2}\right)}{s}\left(\frac{1}{3.6 s+1}\right)
$$

Figure 6 displays the closed-loop responses under nominal condition when subject to 1 unit step change in output disturbance. From the values of IAE, we can clearly notice that the proposed multi-scale control scheme (MSC-PI/P) gives the best performance while the standard single-loop PID control scheme yields the worst performance.

Figure 7 demonstrates the closed-loop responses in the presence of error in time-delay (actual time-delay is 10 units above its nominal value). The Smith predictor scheme exhibits severe oscillatory response (shows almost unstable performance), while both MSC-PI/P and PID control schemes remain stable. Interestingly, just like in the first example, the performance degradation of the proposed multi-scale control scheme is significantly less than of the traditional single-loop 
PID control scheme (increase in the IAE of the former is much less than that of the latter).

Figure 8 illustrates the plot of gain margins of the 3 control schemes versus time-delay modeling error. Unlike the first example, the gain margins for all control schemes increase with the increase in negative value $a$ (actual time-delay is smaller than the model time-delay). Thus, this means that the controllers can become unstable only when the actual timedelay increases beyond certain values above that of nominal value.

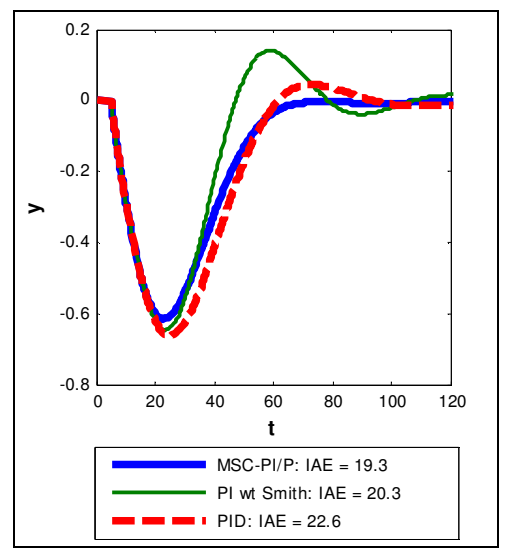

Figure 6. Disturbance rejection performances $a=0$

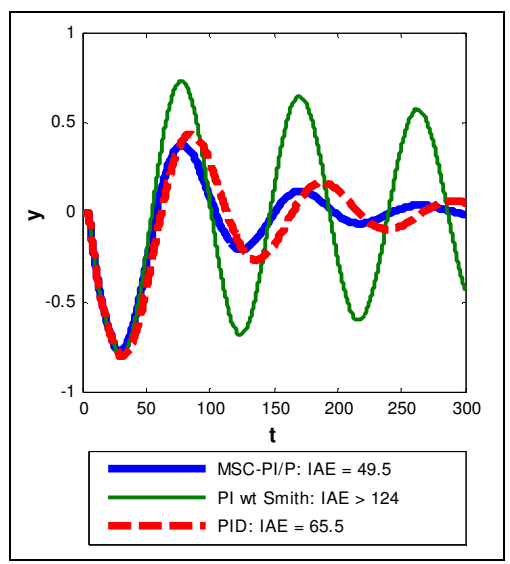

Figure 7. Disturbance rejection performance under plant/model mismatch

$$
(a=10)
$$

From Figure 8, notice that the MSC-PI/P and standard single-loop PID control schemes exhibit similar robustness against time-delay modeling error (both become unstable when the actual time-delay increases by 19.5 units above the nominal value). Meanwhile, the PI controller with Smith predictor can only tolerate modeling error in time-delay up to about 10 units (only about half of that MSC-PI/P). Therefore, like the first example, we can conclude that the proposed multi-scale control scheme can achieve better performance than the conventional PID and PI with Smith predictor schemes. It is interesting to note that, the proposed multi-scale scheme is able to maintain better performance robustness against plant/model mismatch than the Smith predictor scheme.

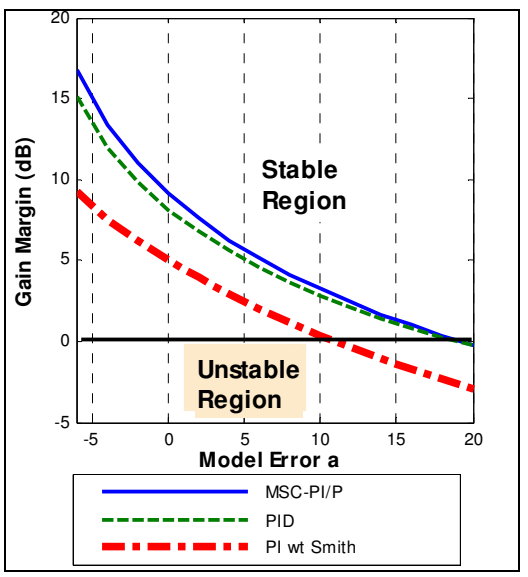

Figure 8. Gain margin versus model error in time-delay

\section{CONCLUSIONS}

In this, paper we have presented a general multi-scale control scheme which can be used to improve the control performance of processes with significant time-delays. Numerical studies and analysis have shown that the proposed multi-scale control scheme is superior over the classical Smith predictor in terms of nominal performance and robustness against time-delay modeling error. It is worthwhile to highlight that, the proposed multi-scale control scheme can provide improved performance over the standard single-loop PID control scheme; surprisingly, this can be achieved by the multiscale control scheme with similar or greater robustness against time-delay modeling error than the standard single-loop PID control scheme.

\section{REFERENCES}

[1] K. J. Astrom, "Limitation on control system performance," Eur. J. Control, vol. 6, no. 1, pp. 2-20, 2000.

[2] O. J. M. Smith, "A controller to overcome dead time," ISA J., vol. 6, no. 2, pp. 28-33, 1959.

[3] Z.J. Palmor, "Stability properties of Smith dead-time compensator controllers," Int. J. Control, vol. 32, no. 6, pp. 937-949, 1980.

[4] T. H. Lee, Q. G. Wang, and K. K. Tan, "Robuts Smith-predictor controller for uncertain delay systems," AIChE J., vol. 42, no. 4, pp. 1033-1040, 1996.

[5] W. Zhang, Y. Sun and X. Xu, "Two degree-of-freedom Smith predictor for processes with time delay," Automatica 34, no. 10, pp. 1279-1282, 1998.

[6] L. Mirkin, Z. J. Palmor, and D. Shneiderman, "Dead-time compensation for systems with multiple I/O delays: a loop-shifting approach," IEEE T. Automat. Contr, vol. 56, no. 11, pp. 2542-2554, 2011.

[7] A. N. Reyes, J. E. N. Rico, C. Bordons, and E. F. Comacho, "A Smith predictive based MPC in a solar air conditioning plant," Journal of Process Control, vol. 15, no. 1, pp. 1-10, 2005

[8] D. E. Seborg, T. F. Edgar, and D. A. Mellichamp, Process dynamics and control, 2nd ed., John Wiley \& Sons, 2003. 